\title{
Die Leitwarte von heute verstehen - die Leitwarte von morgen gestalten!
}

\author{
Tobias Schwarz ${ }^{1}$, Flavius Kehr ${ }^{2}$, Holger Oortmann ${ }^{1}$, Harald Reiterer ${ }^{3}$ \\ Siemens AG, München ${ }^{1}$ \\ Fachbereich Psychologie, Universität Koblenz-Landau ${ }^{2}$ \\ Arbeitsgruppe Mensch-Computer Interaktion, Universität Konstanz ${ }^{3}$
}

\begin{abstract}
Zusammenfassung
In diesem Beitrag wird eine domänenübergreifende Nutzungskontextanalyse vorgestellt, vor dem Hintergrund der Herausforderung einer stetig ansteigenden Komplexität der Mensch-MaschineSchnittstelle in der Arbeitsumgebung von Leitwartenoperatoren. Die mittels teilnehmender Beobachtung und teilstrukturierten Interviews durchgeführte Erhebung in Leitwarten hatte die Zielsetzung Gemeinsamkeiten in Bezug auf demographische, ergonomische und tätigkeitsbezogene Eigenschaften zu identifizieren, sowie Verbesserungspotentiale zu erfragen. Auf der Basis der erhobenen Daten werden neue Möglichkeiten vorgestellt, um die Arbeitsumgebung von Operatoren zu optimieren.
\end{abstract}

\section{$1 \quad$ Einleitung}

Leitwarten, wie sie beispielsweise bei der Energieerzeugung verwendet werden, sind technische Einrichtungen, die Prozesse kontrollieren und steuern (Hollnagel \& Woods 2005). Dabei zählen Tätigkeiten wie Überwachen, Diagnostizieren und Eingreifen zu den Hauptaufgaben eines Operators (Johannsen 1993). Innerhalb der letzten Jahrzehnte stieg durch den erhöhten Grad an Information und die Anzahl der technischen Geräte die Komplexität der Aufgaben eines Operators. Außerdem wird es den Operatoren durch die zunehmende Automatisierung erschwert, ein vollständiges mentales Modell der zu überwachenden Prozesse zu erlangen. Die Generierung eines mentalen Modells ist jedoch essentiell für die Überwachung des aktuellen Systemstatus, im Speziellen beim Feststellen von Veränderungen in der Prozessdynamik (Wickens \& Holland 2000). Zudem lassen sich in Produktionsprozessen zwischen $70 \%$ und $90 \%$ aller Unfälle auf menschliches Versagen zurückführen (Künzler 2002). Menschliche Fehler sind häufig auf die Tatsache zurückzuführen, dass der Mensch mit seinen angeborenen Fähigkeiten beim Entwurf der Systeme nicht ausreichend berücksichtigt wurde. 
Erkenntnisse aus der Kognitionswissenschaft bestätigen, dass eine enge Verbindung zwischen Denkprozessen, der Wahrnehmung und körperlichen sowie sozialen Handlungen besteht (Dourish 2001). Daraus folgt für das Design von interaktiven Systemen, dass der Mensch mit seinen physischen und kognitiven Fähigkeiten, seinem Kontext und seinem sozialen Umfeld zu betrachten ist (Dourish 2001). Eine Lösung für die Problemstellung bietet die Entwicklung nutzer- und arbeitsorientierter Konzepte. Diese zielen darauf ab, Arbeitssysteme ganzheitlich zu gestalten, d. h. der soziotechnischen Tradition folgend die Entwicklung und den Einsatz von Technik, Organisation und Qualifikation der Nutzer gemeinsam zu optimieren. Ziel des Forschungsprojektes „Holistic Workspace“ ist daher die Gestaltung einer holistischen Arbeitsumgebung für Operatoren unter der Berücksichtigung neuer Technologien und Ansätze aus der Mensch-Computer-Interaktion. Leitbild bei der Konzeptentwicklung ist dabei eine Arbeitsumgebung, welche den Operator bei der Ausübung seiner Tätigkeiten und der Einbindung in seine Arbeitsumgebung unter Berücksichtigung seiner Erfahrungen und Fähigkeiten bestmöglich unterstützt. In Anbetracht dessen ist es notwendig, einen aufeinander abgestimmten Ansatz für einen Operatorenarbeitsplatz zu gestalten, der sowohl die technische Infrastruktur (Benutzungsoberfläche, Eingabe-, Ausgabe-, Kommunikationsgeräte), die Arbeitsabläufe als auch die physischen Räumlichkeiten und sozialen Interaktionen berücksichtigt. In diesem Beitrag wird eine Nutzungskontextanalyse über unterschiedliche Domänen vorgestellt, die den Ausgangspunkt des Projektes darstellt. Ziel der Analyse war es, ein Verständnis für die Anforderungen und die Aufgaben sowie für die soziale und physische Umgebung von Operatoren als Nutzer komplexer, sicherheitskritischer Systeme zu entwickeln. Der Beitrag erläutert den Ablauf der Analyse, stellt die bedeutendsten Ergebnisse vor und leitet daraus Grundsätze für die zukünftige Gestaltung von Leitwarten ab. Er schließt mit einem Ausblick, in dem erste Ideen zur Gestaltung einer holistischen Arbeitsumgebung für Operatoren präsentiert werden.

\section{Grundlagen}

In Leitwarten werden die Prozesse von Operatoren nicht mehr direkt am Ort des Geschehens überwacht, sondern zentral auf einer Vielzahl von Bildschirmen. Operatoren übernehmen durch ihre Tätigkeit die Rolle des Prozessführers (Herczeg 2008). Johannsen (1993) und Sheridan (1996) bezeichnen die Arbeitsteilung zwischen Mensch und Maschine als supervisory control. Der Operator nimmt dabei die leitende Kontrolle im automatisierten System ein. Neben Primäraufgaben wie beispielsweise Beobachtung der Prozesse, müssen Operatoren Sekundäraufgaben bearbeiten. $\mathrm{Zu}$ diesen zählen beispielsweise das Führen des Schichtbuches, Abstimmungsgespräche mit Kollegen in der Leitwarte sowie Technikern draußen vor Ort. Größere Störungen treten im Regelfall nur sehr selten ein. Kommt es jedoch zu einem Zwischenfall (anomaler Betrieb), so muss der Operator sofort in der Lage sein, entsprechende Gegenmaßnahmen einzuleiten - denn die Auswirkungen eines Zwischenfalls bergen ein hohes Schadenspotential (Perrow 1984). Durch lange Beobachtungstätigkeiten kommt es zum Abfall der Vigilanz. Die Wahrscheinlichkeit Meldungen zu entdecken, welche auf eine Störung im System hinweisen, sinkt (Johannsen 1993). Boring et al. (2005) weisen darauf hin, dass sich daher für den Usability Engineer in diesem Kontext andere Herausfor- 
derungen stellen als in herkömmlichen Umgebungen. Bei der praktischen Durchführung einer Nutzungskontextanalyse ist mit einigen Herausforderungen zu rechnen:

- Der Fehler- oder Störfall kann aufgrund der geringen Auftrittswahrscheinlichkeit nur erfragt, selten beobachtet werden: „Was würden sie tun wenn ... eintritt“.

- Am Prozess sind größtenteils mehrere Personen beteiligt, die in komplexen Kooperations- und Kollaborationsstrukturen arbeiten.

Aufgrund der Besonderheiten bei der Analyse von komplexen Systemen, im Speziellen für Kraftwerke, wurde von Rasmussen (1986) und Vicente (1999) die Cognitive Work Analysis (CWA) entwickelt. Im Gegensatz zu herkömmlichen Frameworks wird bei der CWA nicht aufgaben-, sondern systemorientiert vorgegangen. Dadurch wird die Modellierung komplexer Systemzusammenhänge vereinfacht.

\section{3}

\section{Untersuchung}

\subsection{Untersuchungsdurchführung und Datenerhebung}

Im Beitrag wird zur Erhebung des Nutzungskontextes eine Mischung aus Aufgaben- und Systemorientierung gewählt, folglich ein Methodenmix aus Cognitive Work Analysis und Contextual Design (Beyer \& Holtzblatt 1998). Die Cognitive Work Analysis bildete den theoretischen Rahmen - die konkrete Anwendung der Methode erfordert jedoch eine langwierige und tiefgreifende Auseinandersetzung mit dem System. Gerade für einen querschnittlichen Vergleich mehrerer Domänen schienen daher die Instrumente des Contextual Design - teilnehmende Beobachtungen und Experteninterviews - besser geeignet. Insgesamt wurden die jeweiligen Domänen zwischen sechs und acht Stunden vor Ort analysiert. Zwei Personen begleiteten die Erhebung, die jeweils im Wechsel die Rolle des Versuchsleiters und die des Protokollanten übernahmen. Diese Vorgehensweise wird von Rosenthal \& Rosnow (2009) empfohlen, um Versuchsleitereffekte zu minimieren. Abbildung 1 gibt einen Überblick über den Ablauf der Untersuchung.

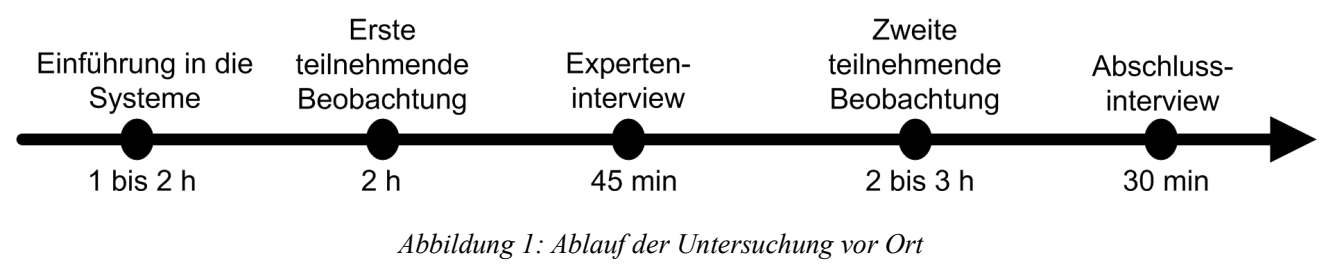

Ziel der ersten und zweiten Untersuchungsphase war es, generische, d.h. nicht auf einen Arbeitsplatz konzentrierte Einblicke zu erhalten. Zu diesem Zweck fanden eine Einweisung in die Systeme sowie eine teilnehmende Beobachtung der Kollaboration- und Kommunikationsstrukturen in der Leitwarte statt. Im Anschluss wurden zwei bis drei Mitarbeitern mit Hilfe eines halbstandardisierten Fragebogens zu demographischen Merkmalen und selbstbe- 
richteter Computeraffinität, zu Arbeitsorganisation, computergestütztem Arbeiten und zum Leitstand allgemein befragt. Die gesammelten Daten und Eindrücke wurden genutzt, um sich in der folgenden teilnehmenden Beobachtung auf Aufgaben und Systeme von bis zu maximal zwei Arbeitsplätzen zu konzentrieren. Hierbei wurden die Teilnehmer auch dazu aufgefordert, laut zu denken (Nielsen 1993). Zum Abschluss der Beobachtungsphase wurde von den Versuchsleitern ein standardisierter Fragebogen ausgefüllt, welcher quantitative Daten über die Leitwarte (z. B. Art und Anzahl von Geräten) erfasste. Um die Validität der Daten sicherzustellen, wurde dieser Fragebogen von beiden Versuchsleitern parallel bearbeitet (Bortz \& Döring 2003). Ein weiteres teilstrukturiertes Interview schloss die Untersuchung ab. Hierbei wurde den Operatoren die Möglichkeit gegeben, über zukünftige Interaktionstechnologien und den möglichen Einsatz in ihrer Arbeitsumgebung zu diskutieren, Vor- und Nachteile ihres derzeitigen Arbeitsplatzes zu erörtern und Wünsche für die Zukunft zu äußern.

\subsection{Ergebnisse}

Insgesamt wurden 6 Leitwarten besucht und analysiert, darunter ein Feuerwehrleitstand, zwei Kraftwerksleitwarten sowie jeweils eine Leitwarte im Tagebau, bei der Flugsicherung und der Postautomatisierung. Es konnten insgesamt 12 unterschiedliche Operatorenarbeitsplätze identifiziert werden, die sich durch einen jeweils anderen Zuständigkeitsbereich und andere Kernaufgaben innerhalb des Gesamtprozesses unterschieden. Die Leitstände waren im Durchschnitt $M=3,48(S D=3,26)$ Jahre alt, der jüngste besuchte Leitstand ein halbes, der älteste 10 Jahre alt. Im Rahmen der Nutzungskontextanalysen wurden Interviews mit insgesamt 13 Mitarbeitern der Leitwarten durchgeführt, sowohl Schichtleiter als auch Operatoren wurden befragt. Tabelle 1 enthält die soziodemographischen Daten dieser Stichprobe. Auffällig sind hierbei das hohe Durchschnittsalter sowie die äußerst heterogenen beruflichen Hintergründe, sowohl bei Operatoren als auch bei Schichtleitern.

\begin{tabular}{|l|l|}
\hline Befragte & 13 männlich (7 Operatoren, 6 Schichtleiter) \\
\hline Durchschnittsalter & 47,38 Jahre $(S D=5,92)$ \\
\hline $\begin{array}{l}\text { Berufsausbildung } \\
\text { Operator }\end{array}$ & $\begin{array}{l}\text { Betriebsschlosser, Feuerwehrmann, Fleischer, Kühlanlagenmonteur, Meister Nachrich- } \\
\text { tentechnik, Industriemechaniker, Verkäufer }\end{array}$ \\
\hline $\begin{array}{l}\text { Berufsausbildung } \\
\text { Schichtleiter }\end{array}$ & $\begin{array}{l}\text { Dipl.-Ing. (FH) Flugsicherung, Kfz-Mechaniker, Kraftwerks-Meister, Logistikfachmann } \\
\text { (2), Stahlbauschlosser }\end{array}$ \\
\hline Computer-Expertise & $3,59(S D=0.92 ;$ Skala von 1 = keine Kenntnisse bis 5 = ausgezeichnete Kenntnisse) \\
\hline
\end{tabular}

Tabelle 1: Soziodemographische Daten der Mitarbeiter

Für einen reibungslosen Ablauf des Prozesses ist eine ständige Kommunikation der beteiligten Personen erforderlich. Entsprechende Nachfragen während der Interviews ergaben, dass nachts und an Wochenenden bis zu 4 Personen gleichzeitig $(M=2,55 ; S D=1,04)$, tagsüber sogar bis zu 8 Personen gleichzeitig $(M=3,73 ; S D=2,24)$ mit demselben System im gleichen Prozess arbeiten. Derzeitige Systeme scheinen diese zwingende Kollaboration aber nur 
wenig zu unterstützen, da meist nur Eingabemöglichkeiten wie Maus und Tastatur zur Verfügung stehen. So zeigte sich im Rahmen der teilnehmenden Beobachtung, dass nach gemeinsam, an einem Arbeitsplatz durchgeführten Teambesprechungen jeder Operator an seinen Arbeitsplatz zurückkehren muss, um die erforderlichen Eingaben vorzunehmen. Kollaboration ist jedoch nicht nur zwischen den Mitarbeitern innerhalb der Leitwarte erforderlich. Auch die Koordination von z. B. Technikern vor Ort stellt eine wichtige Aufgabe dar. Im Normalfall wird per Funk oder Telefon mit diesen Mitarbeitern kommuniziert, häufig muss jedoch für jedes Team von Mitarbeitern ein eigenes Gerät verwendet werden. Im Rahmen der Analyse wurden Sequencemodels (Beyer \& Holtzblatt 1998) erstellt, die zeigen, dass gerade bei anomalem Betrieb die Kommunikation über verschiedene Kanäle parallel stattfinden muss - während z. B. der Techniker vor Ort angefunkt werden kann, ist zur Kommunikation mit der Betriebszentrale ein Handy oder Telefon erforderlich. Hinzu kommt die Kommunikation zwischen den Leitwartenmitarbeitern, die meist von Angesicht zu Angesicht, aber auch indirekt über papierbasierte Artefakte wie Notizen, Memos oder Warnschilder, die einen definierten Zustand ausdrücken, stattfindet. So findet die Verständigung über mehrere Kommunikationskanäle statt. Die vielfältigen digitalen und analogen Kommunikationsmöglichkeiten führen zu einer entsprechenden Zahl an Geräten - bis zu sieben Kommunikationsgeräte wie Telefone, Handys und Funkgeräte befinden sich an einem Arbeitsplatz. Hinzu kommen Eingabe- (Mäuse, Tastaturen, Joysticks etc.) und Ausgabegeräte (Bildschirme, Videomonitore, Großbildschirme etc.), so dass ein durchschnittlicher Leitwartenmitarbeiter bei seiner täglichen Arbeit $M=18,67(S D=8,69)$ Geräte, im Einzelfall bis zu 34 Geräte bedienen und beobachten muss (siehe Abbildung 2).

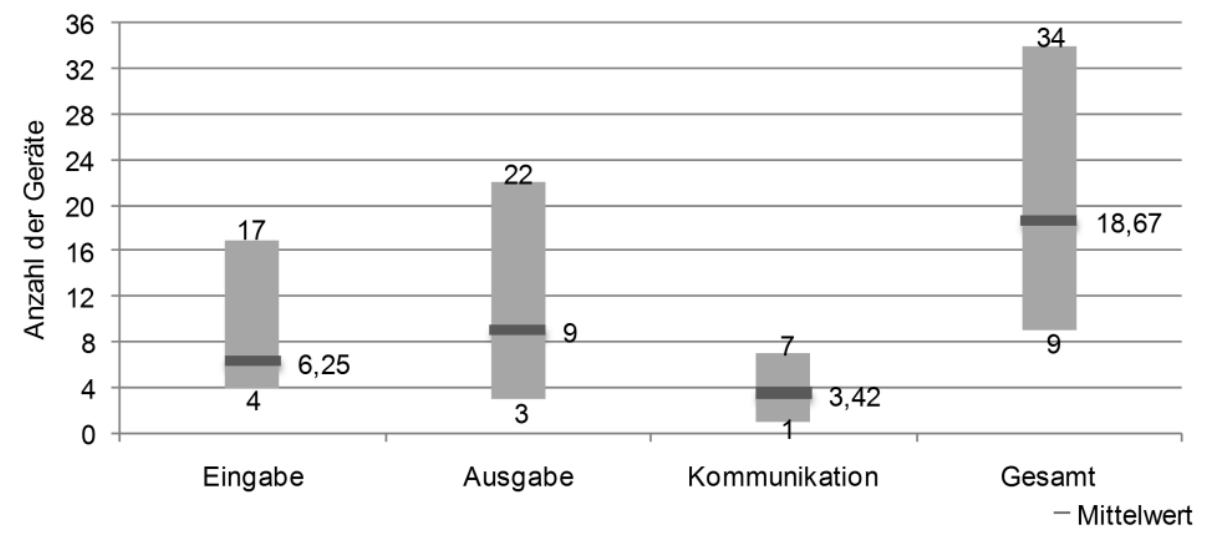

Abbildung 2: Anzahl der Geräte an den Arbeitsplätzen

Ähnlich verhält es sich mit den zu bedienenden Softwareprodukten, wobei hier vor allem Schichtleiter eine große Anzahl unterschiedlicher Produkte beherrschen müssen. Während Operatoren durchschnittlich $M=1,57(S D=2,23)$ Softwareprodukte verwenden, sind Schichtleiter für ihre tägliche Arbeit auf $M=7(S D=1,26)$ Software angewiesen. Dieser Unterschied wird statistisch hochsignifikant $(t(10)=5,5 ; p<.01)$ und zeigt sich besonders deutlich bei Bürosoftware wie Word oder Excel - in beiden Fällen ergibt ein exakter Test 
nach Fisher ein Signifikanzniveau von $p<.01$. Erklärbar wird der Unterschied durch zusätzliche Verwaltungsaufgaben der Schichtleiter, wie das Führen von Schichtplänen. Die hohe Anzahl an unterschiedlichen Geräten und Softwareprodukten ist nach Aussage der Befragten historisch gewachsen. Tatsächlich ließ sich in drei Domänen beobachten, dass zwei oder mehr Softwareprogramme für identische Aufgaben genutzt wurden. In Bezug auf die Informationsvisualisierung der Branchensoftware - vor allem die Darstellung von Meldungen konnte mehrfach beobachtet werden, dass eine Vielzahl von Meldungen beim Operator angezeigt werden (bis zu 12 Meldungen pro Minute). Hinzu kommt der Umgang mit nicht einheitlich gestalteten Farbcodierungen, was auf die unterschiedlichen Softwareprodukte zurückzuführen ist. Ideen für zukünftige Entwicklungen wurden im Abschlussinterview mit den Mitarbeitern $(N=12)$ erörtert. Hierzu wurden neue Interaktionstechnologien vorgestellt und deren Vor- und Nachteile für den spezifischen Arbeitskontext diskutiert. Der mögliche Einsatz der jeweiligen Technologie musste für den eigenen Berufskontext auf einer 3stufigen Skala (Ja, Vielleicht, Nein) eingeordnet werden (siehe Abbildung 3).

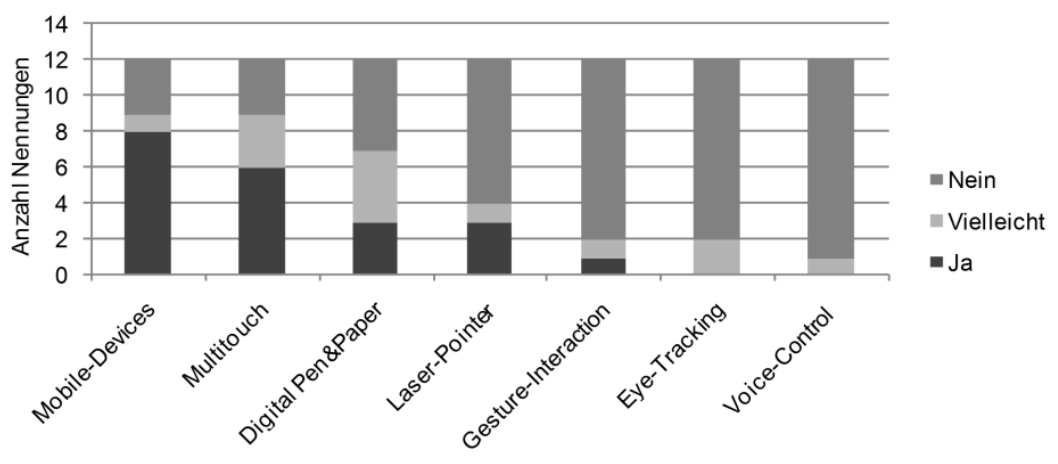

Abbildung 3: Akzeptanz der Interaktionstechnologien

Mobile-Devices würden sich 75\% der Befragten als Ergänzung zu ihren derzeitigen Arbeitsgeräten wünschen, da sich viele Leitwartenmitarbeiter während der Schicht nicht oder nur sehr begrenzt von ihrem Arbeitsplatz entfernen dürfen. Nach Aussagen der Mitarbeiter würde ein mobiles Gerät eine räumliche Flexibilität unterstützen. Die teilnehmende Beobachtung zeigt, dass sich zwei Operatoren vom Arbeitsplatz entfernen müssen, um beispielsweise Systemanzeigen außerhalb der Leitwarte zu überprüfen. Der Workflow der Mitarbeiter kann hier durch den Einsatz eines mobilen Geräts unterstützt werden. Das Gerät könnte beispielsweise jeweils die wichtigsten Meldungen visualisieren. Multitouch-Systeme wurden mit erleichterter Kollaboration und intuitiver Bedienung in Zusammenhang gebracht und daher von 50\% der Befragten als vorteilhaft für den eigenen Arbeitskontext gesehen. Auch einen Einsatz der Digital Pen\&Paper-Technologie konnten sich die meisten Befragten durchaus vorstellen. Die Mitarbeiter zweifelten jedoch an der Alltagstauglichkeit der Geräte. Auf großen Wandbildschirmen wird im Arbeitskontext sehr selten interagiert, diese dienen lediglich zur Überwachung von Teil- oder Gesamtprozessen. Aus diesem Grund wurde der LaserPointer als wenig nützlich beurteilt. Auch Technologien wie Gesture-Interaction, VoiceControl und Eye-Tracking schienen den Befragten bei der Steuerung von Prozessen als we- 
nig dienlich. Über $80 \%$ der Befragten beurteilten die Technologien als nicht hilfreich im Kontext der Leitwarten, da sie als nicht realisierbar schienen. Zum Abschluss wurden die Mitarbeiter nach Optimierungspotentialen an ihren eigenen Arbeitsplätzen befragt. Es ergaben sich 57 Nennungen, die inhaltsanalytisch ausgewertet und aggregiert wurden. Jeweils ein Viertel der Nennungen entfallen auf die Themen physische Arbeitsplatzanordnung/Leitwartengestaltung und digitale Informationsvisualisierung. Bei der Leitwartengestaltung steht besonders der Wunsch nach besseren akustischen, vereinzelt auch Licht- und Klimaverhältnissen im Vordergrund (7 Aussagen). In der Kategorie Informationsvisualisierung wünschen sich die Teilnehmer eine verbesserte Prozessdarstellung (6 Aussagen) und effizientere Anzeigen im Bereich Alarmmanagement (3 Aussagen). Auch scheint der Wunsch nach größeren Bildschirmen mit höherer Auflösung wesentlich zu sein, ebenso die Vereinheitlichung der Geräte in der Arbeitsumgebung. Vereinzelt vermissen die Befragten auch Funktionen zur Personalisierung von Systemen, also z. B. die Möglichkeit, Oberflächen und Einstellungen stärker nach ihren Bedürfnissen zu konfigurieren.

\section{$4 \quad$ Diskussion}

Die Untersuchung hatte das Ziel, die Arbeitsumgebung von Experten aus unterschiedlich komplexen Domänen zu explorieren. Die Ergebnisse liefern wertvolle Erkenntnisse für die Gestaltung eines neuen holistischen Arbeitsplatzes, der den Menschen als Ganzes mit seinen physischen und sozialen Fähigkeiten wahrnimmt. So hat die Analyse gezeigt, dass bei heutigen Entwicklungen immer das einzelne Produkt, nie aber der Mensch im Mittelpunkt der Komposition aller Geräte am Arbeitsplatz steht. Der Operator findet ein Sammelsurium von Geräten vor und muss eine hohe Anzahl an Softwareprodukten beherrschen. Gerade im Störfall, wenn Fähigkeiten zur Entwicklung von Problemlösestrategien gefordert sind (Wittenberg 2000), könnte die hohe Anzahl unterschiedlicher Geräte und Softwareprodukte zu einer kognitiven Überforderung (cognitive overload) führen und fehlerhafte Entscheidungen nach sich ziehen (Grams 1998). Gerade in komplexen Situationen kann es beispielsweise leicht zu Verwechslungen der Eingabegeräte kommen. Ziel eines ganzheitlichen Konzepts für Leitwarten sollte daher sein, Geräte und Softwareprodukte auf ein Minimum zu reduzieren sowie eine konsistente Benutzungsoberfläche und ein durchgängiges Bedienkonzept zu gestalten, um einen cognitive overload der Operatoren zu vermeiden. Wie den Aussagen der Probanden zu entnehmen ist, spielt die physische und soziale Arbeitsumgebung eine wichtige Rolle und beherrscht maßgeblich die kognitiven Prozesse während des gesamten Arbeitsprozesses. Als ein erster Ansatz scheint das Verändern der physischen Umgebung erfolgversprechend. Die Arbeitsplatzanordnung oder die Leitwartengestaltung stellen nur zwei Beispiele dar. Zukünftige Entwicklungen sollten jedoch auch kollaborative Arbeitsabläufe stärker unterstützen. Die Analyse hat gezeigt, dass die Kollaboration innerhalb der Leitwarte und Kommunikation mit Mitarbeitern vor Ort essentielle Bestandteile alltäglicher Arbeitsabläufe sind, welche von den derzeitigen Technologien aber nur unzureichend unterstützt werden. Gerade für kollaborative Arbeit sind die vorherrschenden Interaktionsmodalitäten per Maus und Tastatur nicht optimal geeignet. Körperliche und soziale Faktoren, die für die Arbeit essentiell sind, werden hier vernachlässigt. Die bestehenden Geräte und Anwendungen verhindern es flexi- 
bel in verschiedenen Situationen zu reagieren. Soziale Interaktionen zwischen Operatoren werden jedoch auch häufig über indirekte Kommunikation in Form von Artefakten in der realen Welt (z. B. Schichtbücher, Schichtpläne, Checklisten, Notizen) ausgedrückt. Diese Art der impliziten Kommunikation ist vor allem für übergeordnete Aktivitäten wie Koordination und Kommunikation der Operatoren wichtig und wird häufig nicht hinreichend unterstützt. Auch die Informationsvisualisierung und das Meldungsmanagement innerhalb der Softwaresysteme tragen wenig dazu bei, die Operatoren kognitiv zu entlasten - stattdessen werden häufig alle eingehenden Meldungen angezeigt, so dass letztlich der Operator über Wichtigkeit und Priorität einzelner Meldungen entscheiden muss. Des Weiteren können eingehende Meldungen nicht durchgängig an verschiedensten Orten abgerufen werden, was das Arbeiten abseits des Arbeitsplatzes nicht ermöglicht. Folglich kann es bei Abläufen wie beispielsweise der Schichtübergabe zu Unterbrechungen kommen. Diese Problematik wird auch von den Leitwartenmitarbeitern gesehen, die Informationsvisualisierung und Vereinheitlichung von Geräten am Arbeitsplatz als Optimierungspotentiale erkennen. Zukünftige Entwicklungen im Leitwartenkontext sollten sich daher stärker um einheitliche und nachhaltige Konzepte bemühen, die auf die kognitiven Informationsverarbeitungskapazitäten aber auch auf die sozialen und physischen Fähigkeiten der Operatoren zugeschnitten sind. Die Experteninterviews haben weiterhin gezeigt, dass der Einsatz neuer Technologien von den Operatoren akzeptiert werden würde. Es gilt jedoch zu beachten, dass es sich bei den befragten Operatoren über alle Leitwarten hinweg um Männer mittleren Alters handelte, die aus sehr unterschiedlichen Berufen stammen. Beide Faktoren stellen, trotz der hohen Selbsteinschätzung der Probanden bezüglich ihrer Computerexpertise, besondere Anforderungen an die Gestaltung.

\section{$5 \quad$ Ausblick}

Im Folgenden werden erste Ideen unter Berücksichtigung der erlangten Erkenntnisse zur Umsetzung einer holistischen Arbeitsumgebung beschrieben. Da in der Untersuchung beobachtet werden konnte, dass die Interaktion und Kommunikation einerseits in der realen Welt (z. B. Diskussionen, Papier, Checklisten) und andererseits über digitale Systeme (z. B. Meldungen, Statusüberwachung) stattfindet, spielt die Vermischung der beiden Ebenen in den zu entwickelnden Konzepten eine entscheidende Rolle. Das aus der Mensch-ComputerInteraktion stammende Paradigma Reality-Based Interaction von Jacob et al. (2008) bietet hierfür eine Orientierungshilfe. In diesem Paradigma wird vorgeschlagen, die Interaktion zwischen Mensch und Computer an der realen Welt zu orientieren, um gelernte und evolutionsbedingte Charakteristiken und Verhaltensweisen des Menschen zu nutzen. Ziel ist dabei, durch gezielten Einsatz neuer technologischer Möglichkeiten der computergestützten Interaktion und Kommunikation, Menschen in ihren realen Abläufen zu entlasten. Unabdingbar ist dabei der nahtlose Wechsel zwischen realweltlicher sowie computergestützter Interaktion. Die Gestaltung der Arbeitsumgebung sollte sich jedoch nicht allein auf die Interaktion zwischen Mensch und Computer beschränken. Die Kommunikation zwischen den Operatoren, die Arbeitsabläufe sowie die Gestaltung des physischen Arbeitsumfelds sollten gleichermaßen beachtet werden. Abbildung 4 zeigt erste Ideen zur Umsetzung der Leitwarte der Zukunft. Neue Interaktionstechnologien, insbesondere die Multitouch-Technologien, ermögli- 
chen simultane Eingaben mehrerer Personen und fördern damit kollaboratives Arbeiten. Durch die direkte Manipulation wird zugleich die Bedienung natürlicher. Weiterhin kann durch eine größere Visualisierung, zum Beispiel an großen Wandbildschirmen, kollaboratives Arbeiten bei Lagebesprechungen und Schichtübergaben unterstützt werden. Die Wandbildschirme könnten zusätzlich mit der sog. Ambient Light Technologie ausgestattet werden und beispielsweise je nach Status eine andere Farbgebung der Hintergrundbeleuchtung annehmen. So könnte sich im Störfall die Umrandung beispielsweise rot einfärben. Das neue Konzept einer holistischen Arbeitsumgebung sieht weiterhin vor, die Arbeitsabläufe durch ein mobiles Gerät (z. B. iPhone) zu unterstützen. Die Visualisierung der wichtigsten Meldungen auf diesen Geräten ermöglicht es, Aufgaben durchgängig an verschiedensten Orten auch abseits des eigenen Arbeitsplatzes verantwortungsvoll wahrzunehmen.

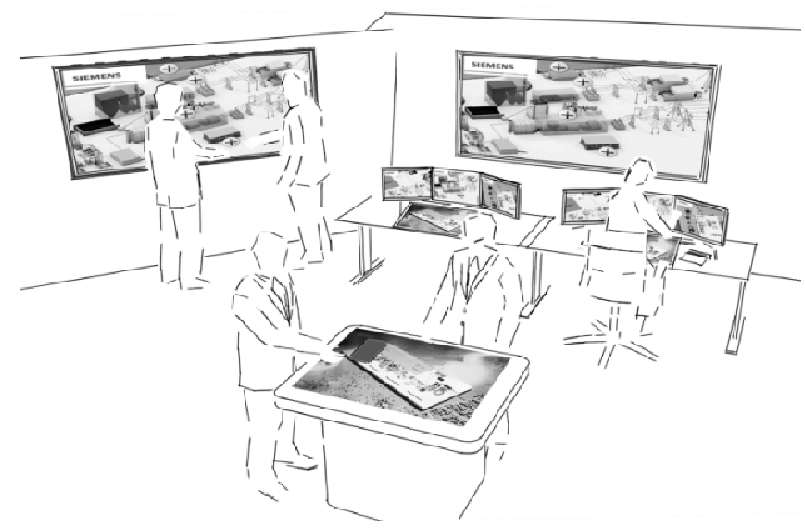

Abbildung 4: Die Idee der holistischen Arbeitsumgebung

Die Führung von Protokollen und Schichtplänen wird mittels Digital Pen\&PaperTechnologie durchgeführt, wodurch die Daten sowohl in analoger als auch in digitaler Form vorliegen. Somit findet ein nahezu nahtloser Übergang zwischen digitaler Welt und physischem Arbeitsbereich statt. So können andere Teammitglieder von ihrem Arbeitsplatz aus jederzeit auf Protokolle oder Schichtpläne zugreifen, diese drucken oder mittels Suchfunktion in den Dokumenten suchen. Alle Daten werden mit einem Zeitstempel in einer Datenbank gespeichert, entsprechende Änderungen werden dokumentiert und können jederzeit nachvollzogen werden. Zusätzlich können die Daten mit Memos angereichert werden, um so eventuelle Sprachnotizen im Kontext zu hinterlassen. Auf Basis der Erkenntnisse aus den domänenübergreifenden Untersuchungen werden im nächsten Schritt erste Konzepte der Vision einer holistischen Arbeitsumgebung für Operatoren umgesetzt. Dabei soll, wie in den ersten Ideen beschrieben, ein nahtloser Wechsel zwischen der realweltlichen und computergestützten Interaktion sowie Kommunikation möglich sein.

\section{Literaturverzeichnis}

Beyer, H. \& Holtzblatt, K. (1998). Contextual design: defining customer-centered systems. San Francisco: Morgan Kaufmann Publishers Inc. 
Boring, R. L., Hugo, J., Richard, C. M., \& Dudenhoeffer, D. (2005). The Role of Human-Computer Interaction in Next-Generation Control Rooms. CHI 2005 - Special Interest Groups SIGs. New York: ACM, S. 2033-2034.

Bortz, J. \& Döring, N. (2003). Forschungsmethoden und Evaluation für Human- und Sozialwissenschaftler. 3. Auflage. Berlin: Springer.

Dourish, P. (2001). Where The Action Is: The Foundations of Embodied Interaction. Cambridge: MIT Press.

Grams, T. (1998). Bedienfehler und ihre Ursachen (Teil 1). Automatisierungstechnische Praxis atp 40 (3). München: Oldenbourg Verlag, S. 53-56.

Herczeg, M. (2008). Vom Werkzeug zum Medium: Mensch-Maschine-Paradigmen in der Prozessführung. In Grandt, M. \& Bauch, A. (Hrsg.): Beiträge der Ergonomie zur Mensch-System-Integration, DGLR-Bericht 2008-04/01. Bonn: Deutsche Gesellschaft für Luft- und Raumfahrt.

Hollnagel, E. \& Woods, D. (2005). Joint cognitive systems. Foundations of Cognitive systems engineering. Boca Raton: Taylor \& Francis.

Jacob, R. J., Girouard, A., Hirshfield, L. M., Horn, M. S., Shaer, O., Solovey, E. T., \& Zigelbaum, J. (2008). Reality-based interaction: a framework for post-WIMP interfaces. Proceeding of the twenty-sixth annual SIGCHI conference on Human factors in computing systems CHI 2008. New York: ACM, S. 201-210.

Johannsen, G. (1993). Mensch-Maschine-Systeme. Berlin: Springer.

Künzler, C. (2002). Kompetenzförderliche Sicherheitskultur, Ganzheitliche Gestaltung risikoreicher Arbeitssysteme Mensch, Technik, Organisation. Band 36. Zürich: vdf Hochschulverlag.

Nielsen, J. (1993). Usability Engineering. Boston: Academic Press.

Perrow, C. (1984). Normal accidents. Living with high-risk technologies. New York: Basic Books.

Rasmussen, J. (1986). Information Processing and Human-Machine Interaction. New York: NorthHolland.

Rosenthal, R. \& Rosnow, R. L. (2009). Artifacts in Behavioral Research. New York: Oxford University Press.

Sheridan, T. B. (1996). Speculations on future relations between humans and automation. In Parasuraman, R. \& M. Mouloua M. (Hrsg.): Automation and Human Performance - Theory and Applications. Mahwah: Lawrence Earlbaum Associates, S. 449-460.

Vicente, K. J. (1999). Cognitive Work Analysis: Towards Safe, Productive, and Healthy ComputerBased Work. Hillsdale: L. Erlbaum Associates Inc.

Wickens, C. D. \& Holland, J. G. (2000). Engineering psychology and human performance. New Jersey: Prentice Hall.

Wittenberg, C. (2000). Virtuelle Prozessvisualisierung am Beispiel eines verfahrenstechnischen Prozesses. Düsseldorf: VDI.

\section{Kontaktinformationen}

Tobias Schwarz (Siemens AG)

Telefon: +49 (89) 636-49653, E-Mail: schwarz.tobias.ext@siemens.com 